\title{
Generation of Precise Wide-Area Geocoded Elevation Models with ERS SAR Data
}

\author{
Marc BARA, Oscar MORA, Miquel ROMERO, Antoni BROQUETAS
}

\section{Universitat Politècnica de Catalunya (UPC). Campus Nord, C/ Jordi Girona, 1-3, 08034 Barcelona, Spain. Phone / Fax: +34 934011065 / +34 93401 7232. E-mail: mabara@voltor.upc.es}

\section{ABSTRACT}

In this paper we present an improved technique for the generation of Digital Elevation Models (DEM), capable of dealing with full scene images $(100 \times 100 \mathrm{~km})$ coming from an interferogram obtained with ERS satellite data. Starting from an interferometric processor aimed to the geocoding of smaller areas, now we expound the new improvements based on the use of Ground Control Points (GCP) in order to calibrate some imprecisions which appear in the case of very wide swaths. A generated DEM of the test zone of Tarragona (Spain) and its error assessment are presented.

\section{INTRODUCTION}

The most popular application of satellite SAR interferometry is the generation of high-quality Digital Elevation Models. In order to perform this work, an accurate phase to height conversion as well as an exact geocoding algorithm are required.

The basis of the interferometric process has been widely described in many papers: image registration, range filtering, interferogram generation, flat Earth correction, fringe filtering and phase unwrapping [2]. After phase unwrapping the interferometric process provides a phase image which has to be converted into height information and geocoded to a standard cartographic system. This is basically a geometric problem which requires a precise knowledge of both the orbit and the true Earth shape (the local datum).

In this paper we remark the importance of precise geometry and timing knowledge in order to minimise the errors when generating geocoded images: a technique which makes use of GCP to refine this information is presented. The usefulness of this methodology to generate wide-area maps has been checked with a full ERS tandem scene.

\section{THE ORBIT}

In order to calculate the satellite position for each range line in the image, the 'Precise Orbits' files of D-PAF have been used. In the precise orbit file, the state vectors are spaced $30 \mathrm{~s}$ $(225 \mathrm{~km})$ and the area covered by the SLC image is around $100 \mathrm{~km}$. Thus, it is necessary to interpolate the data from the two nearest ephemerids [1][3]. The problem is that the achieved precision (15 cm in radial direction) is not enough for avoiding systematic errors due to the lack of precision in baseline. Therefore, additional information to refine both orbital positions is needed.
In this paper we propose the use of GCP on the studied surface in order to calibrate the orbits. If the SAR image is available, it is possible to find several well-known points (like road crossings, lakes, etc.) in the amplitude image and their corresponding height values on the local datum, using other mapping sources. Figure 1 shows the process to know the satellite positions with an accuracy of few $\mathrm{cm}$. It is divided in two parts: in a first step, the orbits are calculated with the PRC files provided by D-PAF; in a second part, starting from the calibration made with GCP, the orbits are calculated in a much more precise way.

\section{GEOCODING}

A geocoding process [4] consists of three fundamental steps:

- Flat Earth removing.

- Phase to height conversion.

- Ground range projection.

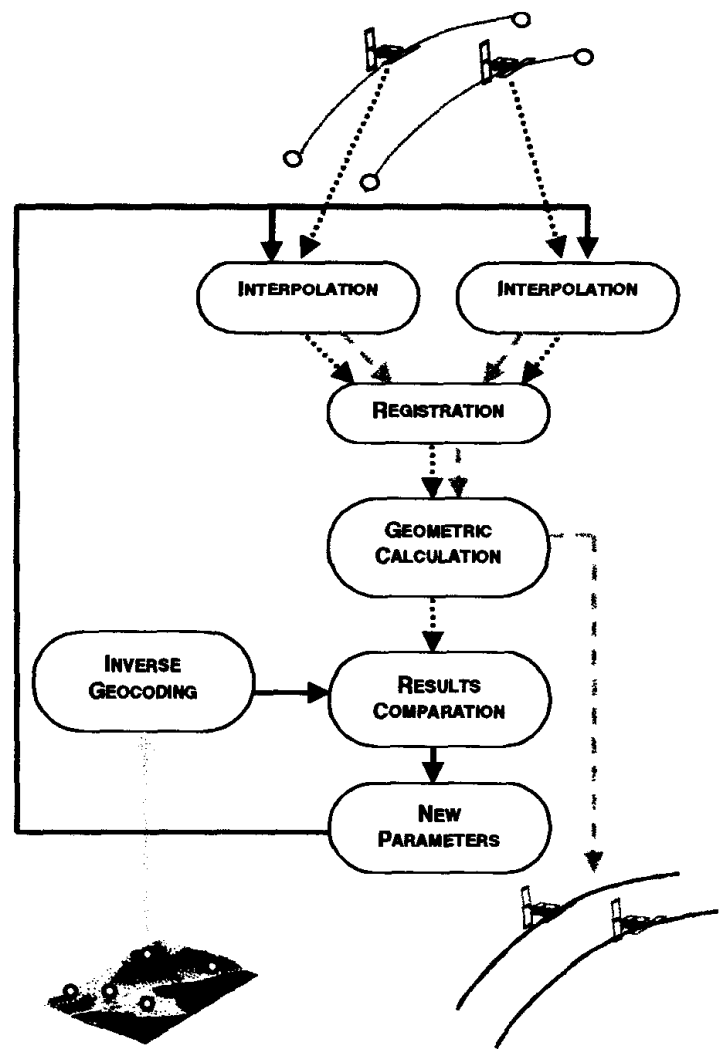

Figure 1. Orbit generation layout 
The first step demands an accurate knowledge of the azimuth time of the satellite above the studied area. Indeed, the subtraction of the flat Earth term is carried out over the reference ellipsoid (Ellipsoidal Earth term), which is characterised by a variable curvature. Therefore, it is necessary to know the 'Near Range Zero Doppler Time' very precisely. As an example, with a baseline of $100 \mathrm{~m}$, a mistake of $1 \mu \mathrm{s}$ in the 'Near Range Zero Doppler Time' yields an error of about $100 \mathrm{~m}$ in the range direction. The implemented method of Fig. 1 offers a solution for this kind of error, since it is capable of correcting the times provided in the SLC headers to obtain accurate orbits. Then, the quality of the orbits is maximised and, consequently, the flatEarth subtraction (see Fig. 2) does not introduce additional distortions.

The second step consists of converting phase to height in slant-range coordinates. This process is carried out accurately with a high order conversion described in [1][3], using the range, Doppler and ellipsoid equations.

However, after completing the conversion, some systematic phase errors can be observed when geocoding wide areas, which lead to height slopes in range and azimuth directions. They appear basically as a result of the atmospheric propagation and the wrong baseline calculation for each range line. In order to implement a second adjustment of the wave paths to both antenna positions, we propose the use of GCP, which can be useful to estimate these systematic inaccuracies. Then, with this new phase, the process is repeated to get highprecision height information.

The third step is oriented to the conversion of the height information from slant-range coordinates onto a standard cartographic reference system, like UTM. In this way, we dispose of precise DEM which can be integrated, for instance, into a GIS.

\section{RESULTS WITH ERS DATA}

Using this technique we have geocoded the area of Tarragona (Spain) with data collected by ERS-1 and ERS-2 with a 100-m baseline. This zone is quite heterogeneous, with coastline, the Ebro river, and a wide range of topographic features from the flat area in Delta del Ebro to the mountains in the inland up to $1200 \mathrm{~m}$. Moreover, some atmospheric artifacts can be observed at Delta del Ebro which yield important height errors, as it is shown in the next section.

The phase to height conversion has been performed using 14 control points spread over the image. Finally, the map has been geocoded to a UTM grid with a 30 -m spacing. Figure 3 shows a $3 \mathrm{D}$ rendering of the wide-area DEM.

\section{ERROR ASSESSMENT}

In this section we present a comparison between the obtained DEM with a high accuracy reference provided by the Institut Cartogràfic de Catalunya (ICC).

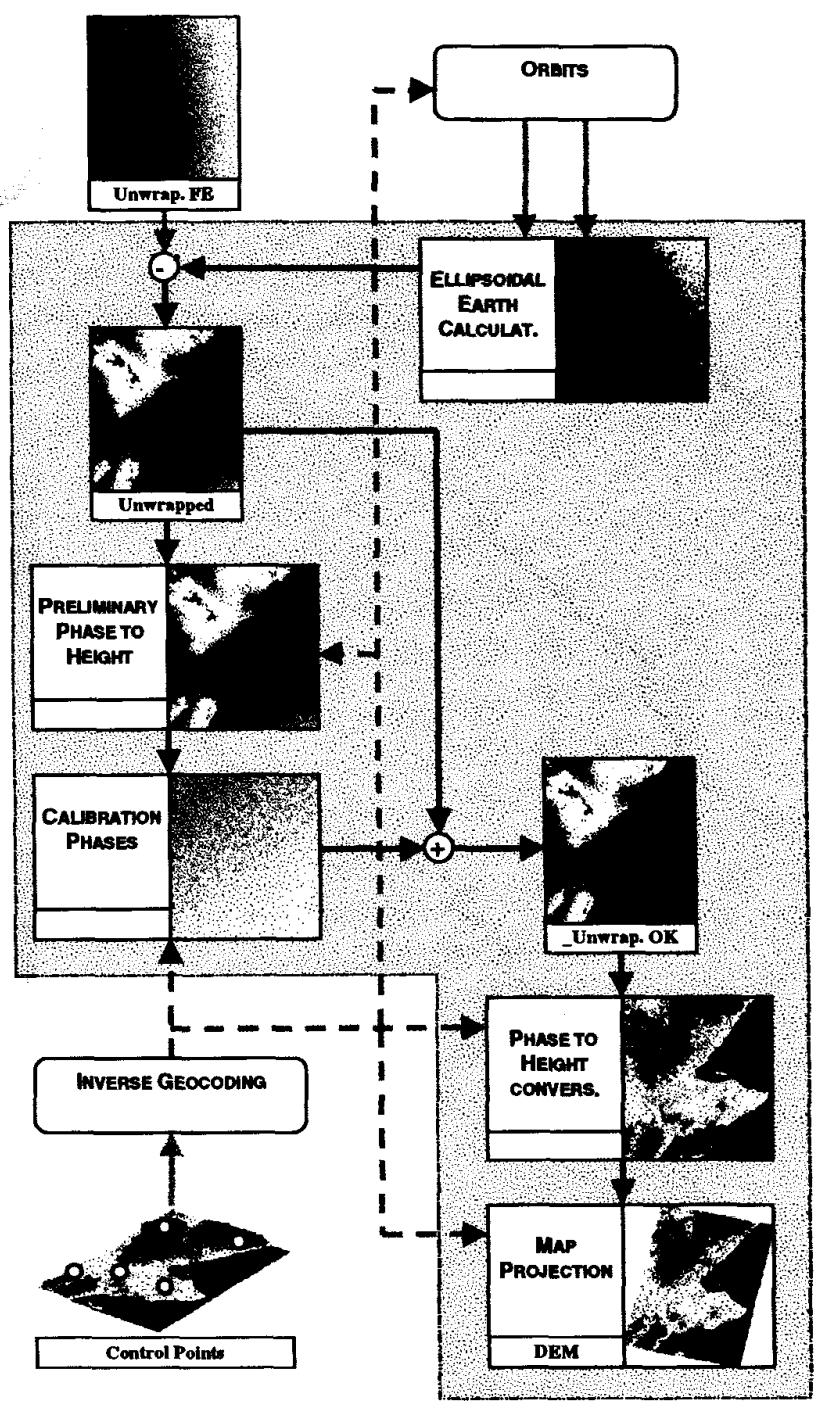

Figure 2. Geocoding layout

The reference DEM has a $2 \mathrm{~m}$ rms vertical accuracy and a $30 \mathrm{~m}$ horizontal grid spacing.

To perform a detailed study, we have selected a smaller area which is affected by different kind of errors, since it comprises the Delta del Ebro (flat zone) and some mountains. The result is shown in Fig. 4. As we can see, there are some atmospheric artifacts in the flat area, as well as shadowing and layover phenomenons in the mountainous area. They produce the most significant errors in the obtained DEM. For example, the atmospheric artifacts at Delta del Ebro generate hills up to $100 \mathrm{~m}$, while in the shadowed zones of the mountains the error reaches the value of $240 \mathrm{~m}$.

However, this kind of inaccuracies can not be attributed to the interferometric process, since they come from the acquisition geometry itself. 


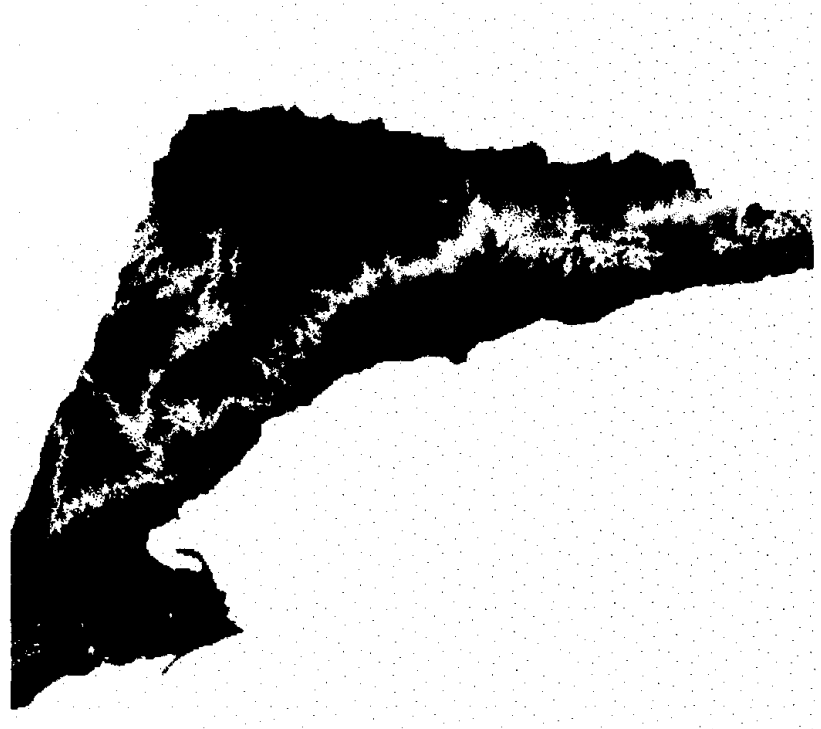

Figure 3. 3D perspective view of the obtained DEM

The geocoding process provides the highest precision in the favourable zones of the interferogram. For instance, in Delta del Ebro we have obtained a rms error of approximately 16 $\mathrm{m}$. In the mountains, as a result of shadowing and layover, this value degrades to $56 \mathrm{~m}$. Indeed, we can classify two different type of errors:

Errors associated to rough topography: Interferometric coherence is degraded due to the high slopes of the terrain. Moreover, the topography itself makes difficult the interpolation where less data is available.

Errors associated to atmospheric artifacts: These artifacts are the responsible for the generation of small hills up to a height of $100 \mathrm{~m}$ in Delta del Ebro.

\section{CONCLUSIONS}

When geocoding large interferograms, the exact knowledge of satellite geometry and time parameters is extremely important. Orbit precisions of centimetres and time accuracies of nanoseconds are required. In this paper we have shown an operative technique to refine the initial supplied orbit parameters

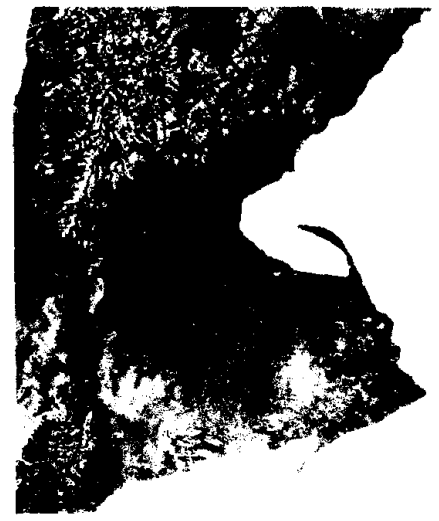

Figure 4. Error between the InSAR DEM and the ICC reference DEM.

These new parameters, added to the precise correction of the ellipsoidal-Earth term, a high order algorithm of phase to height conversion and the support of control points allow the generation of accurate wide-area DEMs from ERS SAR data without the appearance of systematic errors. The methodology has been checked with real data, and a detailed error assessment has been presented in order to discuss the performance of satellite repeat-pass interferometry.

\section{ACKNOWLEDGMENT}

The authors would like to thank the CICYT (Spanish Commision for Science and Technology) and the CIRIT (Catalan Commission for Research) for the financial support of this work. They also acknowledge the ICC (Cartographic Institute of Catalonia) for providing the DEM used for the error assessment.

\section{REFERENCES}

[1] D. Carrasco, D. Esteban, F. Lopez, J. Tena, O. Rodríguez, M. Bara, A. Broquetas, "Precise geometry techniques for ERS SAR Interferometry", European Conference on Synthetic Aperture Radar, EUSAR'98, 1998.

[2] F. Rocca, "An overview of ERS SAR interferometry", Proc $3^{\text {rd }}$ ESA ERS Symposium, Florence, May 1997.

[3] D. Carrasco, "SAR Interferometry for digital elevation model generation and differential applications", $\mathrm{PhD}$ Dissertation, March 1998.

[4] G. Schreider, "SAR Geocoding: Data and systems", Wichmann ed. 1993 